\title{
CW lodine Ion Laser Excited by an Electron Beam
}

\author{
J. D. MEYER, J. J. ROCCA, Z. YU, AND G. J. COLLINS
}

Abstract-CW laser oscillation has been obtained on the $\mathbf{5 7 6 0 . 7}$ and $6127.5 \AA$ transitions of singly ionized iodine in an $\mathrm{He}-\mathrm{I}_{2}$ plasma excited by a dc electron beam.

$\mathbf{R}^{\mathrm{R}}$ ECENTLY, we proposed [1] the use of a dc electron beam created plasma as a new active medium for $\mathrm{CW}$ ion lasers. We report in this paper the generation of $\mathrm{CW}$ laser oscillation in a $\mathrm{He}-\mathrm{I}_{2}$ gas mixture using electron beam pumping. $C W$ laser radiation was obtained on the $5760.7 \AA\left(6 p^{13} D_{2}-\right.$ $\left.6 s^{13} D_{1}^{o}\right)$ and $6127.5 \AA\left(6 p^{13} D_{1}-6 s^{13} D_{2}^{o}\right)$ transitions of singly ionized iodine.

This is the first time that a CW iodine ion laser has been obtained using electron beam excitation. The $6 p^{13} D_{2}$ and $6 p^{13} D_{1}$ iodine ion levels are 0.23 and $0.51 \mathrm{eV}$ below the $\mathrm{He}^{+}$ ground state at $24.58 \mathrm{eV}$. Thermal energy charge transfer reactions between ground state helium ions and iodine atoms have a cross section of $1.5 \times 10^{-15} \mathrm{~cm}^{2}$ [2]. It seems likely that charge transfer is the major excitation mechanism of upper laser levels of the $\mathrm{He}-\mathrm{I}^{+}$electron beam laser, as it also occurs in the He- $\mathrm{I}^{+}$hollow cathode laser [3], and in the $\mathrm{He}-\mathrm{I}^{+}$ positive column laser [4]. However, direct electron impact excitation may be significant.

We believe the electron beam excitation scheme demonstrated here in $\mathrm{He}-\mathrm{I}^{+}$can be extended to a large number of laser systems using thermal energy charge transfer reactions of the types $\mathrm{He}^{+}+M \rightarrow \mathrm{He}+M^{+*}+\Delta E$, in which $M^{+*}$ denotes the upper laser level of a metal ion and $\Delta E$ is the energy defect. The experimental setup used to obtain laser radiation from singly ionized iodine is shown in Fig. 1 .

The electron beam is generated by a glow discharge in a chamber that is separated from the plasma tube by a water cooled iodine trap connected to the vacuum pump. In this way, the concentration of iodine in the active medium, and the electron beam discharge parameters can be independently optimized. Our glow discharge electron guns produce a wellcollimated electron beam of an energy between 1 and $10 \mathrm{keV}$ and a current up to $1 \mathrm{~A}$. These electron guns have two unique features that make them especially useful for exciting $\mathrm{CW}$ ion lasers. The first is their capability of operating in helium at a pressure up to 3 torr without differential pumping. The second is a clear optical path through the electron gun axis that permits one to match the electron beam created plasma volume with the corresponding volume of an optical resonator without the need of a magnetic coil to deflect the electron beam.

Manuscript received November 16, 1981. This work was supported by the National Science Foundation.

The authors are with the Department of Electrical Engineering, Colorado State University, Fort Collins, CO 80523.

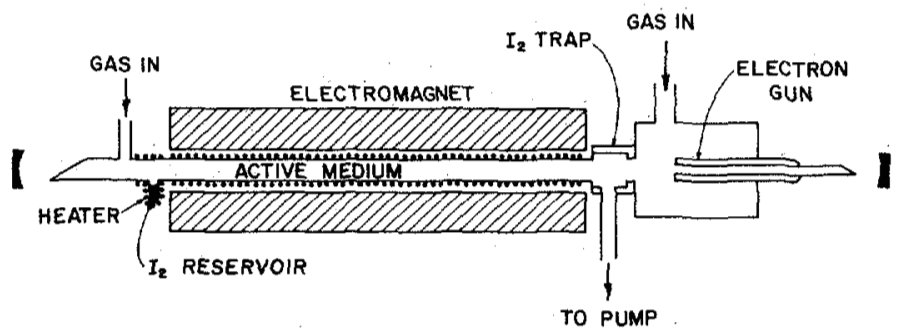

Fig. 1. Schematic diagram of the electron beam excited iodine laser.

The stainless steel plasma tube is $1.1 \mathrm{~cm}$ in diameter and $120 \mathrm{~cm}$ in length. A solenoid $100 \mathrm{~cm}$ long, capable of producing a magnetic field of strength up to $4.2 \mathrm{kG}$ surrounds the plasma tube. The axial magnetic field helps to efficiently deposit the electron beam energy into the plasma, keeping the beam well collimated, and thereby reducing the loss of energetic electrons to the walls of the plasma tube. An electric heater surrounds the plasma tube to avoid condensation of $I_{2}$ on the walls when the laser is operated at low electron beam discharge powers.

The $I_{2}$ reservoir (see Fig. 1) is independently heated and the temperature controlled within $\pm 1^{\circ} \mathrm{C}$. The iodine vapor is distributed in the plasma tube by flowing helium at rates between 250 and 550 standard cubic centimeters per minute (sccm). A helium flow of $400 \mathrm{sccm}$ corresponds to an average helium pressure in the tube of 2 torr. Brewster angle windows and an external resonator consisting of a $2 \mathrm{~m}$ broad-band total reflector and a $3 \mathrm{~m}$ output coupler were used. The output coupler employed has a 0.2 percent transmission at $6150 \AA$ and a transmission of $<1$ percent at $5770 \AA$. Fig. 2(a) shows the dependence of the $5760.7 \AA$ laser line output intensity upon electron beam discharge voltage and current for a magnetic field strength of $3.9 \mathrm{kG}$ and an iodine sidearm termperature of $45^{\circ} \mathrm{C}$. The optimum value of the magnetic field strength was observed to increase as the electron beam discharge current and voltage increase. Fig. 2(b) and (c) show the variation of the output laser intensity as a function of sidearm temperature and helium flow, respectively. Fig. 3(a)-(c) shows the dependence of the $6127.5 \AA$ line upon the same parameters. As shown by Figs. 2(a) and 3(a), the laser output intensity for both lines is a sharply rising function of discharge current beyond threshold and continues to rise up to the highest current investigated. Absolute laser output power has not been measured. The lowest observed threshold for the 5760.7 and $6127.5 \AA$ lines were 380 and $390 \mathrm{~mA}$, respectively.

In summary, we have obtained $\mathrm{CW}$ laser radiation from singly ionized iodine using electron beam pumping. Since the elec- 


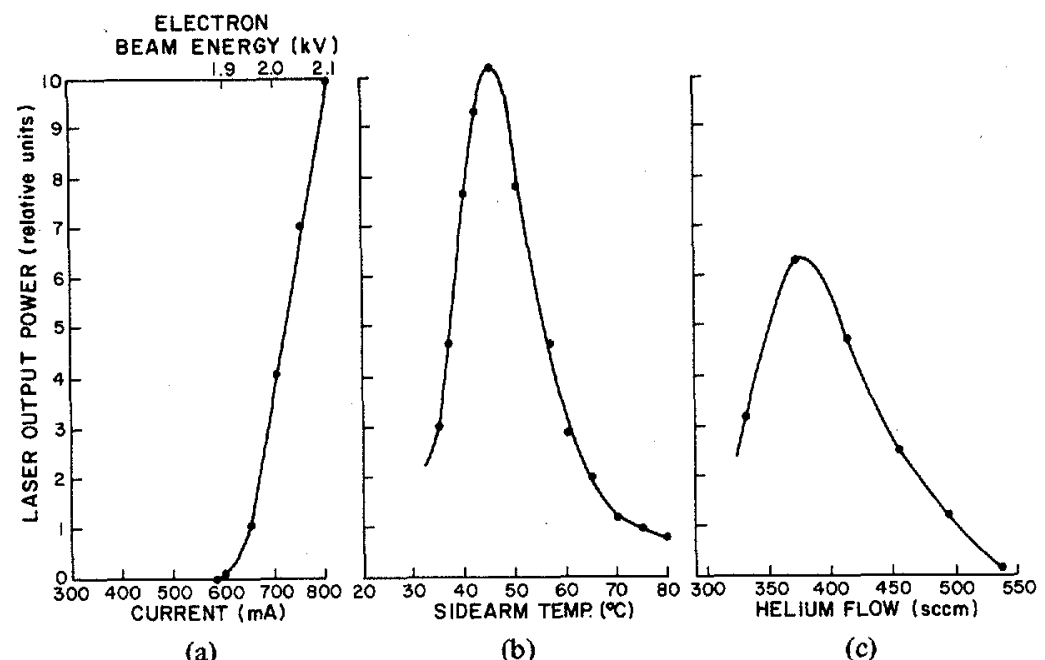

(a)

(b)

(c)

Fig. 2. Behavior of the $5760.7 \mathrm{~A}$ line of singly ionized iodine. (a) Laser output as a function of electron beam discharge current and energy. Sidearm temperature is $45^{\circ} \mathrm{C}$. Magnetic field strength is $3.9 \mathrm{kG}$. Helium flow is $400 \mathrm{sccm}$ corresponding to an average He pressure in the plasma tube of 2 torr. (b) Laser output power as a function of sidearm temperature. Magnetic field strength is $3.9 \mathrm{kG}$. Helium flow is $400 \mathrm{sccm}$. (c) Laser output power as a function of helium flow. Current is $700 \mathrm{~mA}$. Magnetic field strength is $3.75 \mathrm{kG}$. Sidearm temperature is $42^{\circ} \mathrm{C}$.

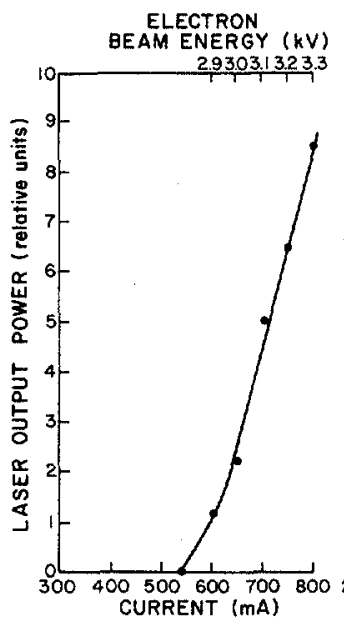

(a)

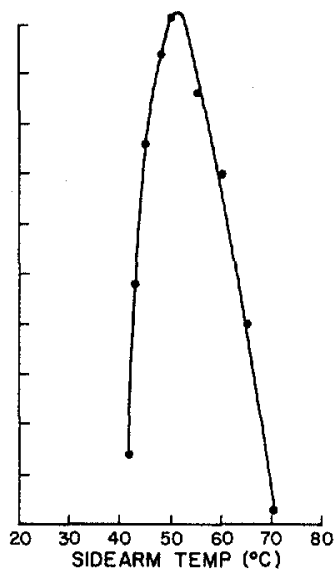

(b)

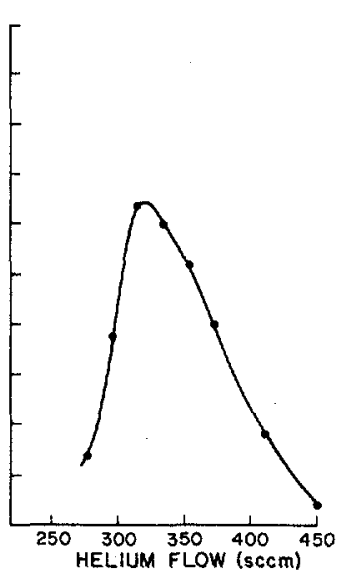

(c)

Fig. 3. Behavior of the $6127.5 \AA$ line of singly ionized iodine. (a) Laser output as a function of electron beam discharge current and energy. Sidearm temperature is $51^{\circ} \mathrm{C}$. Magnetic field strength is $3.9 \mathrm{kG}$. Helium flow is $310 \mathrm{sccm}$ corresponding to an average He pressure in the plasma tube of 1.6 torr. (b) Laser output power as a function of sidearm temperature. Magnetic field is $3.9 \mathrm{kG}$. Helium flow is 310 sccm. (c) Laser output power as a function of helium flow. Current is $700 \mathrm{~mA}$. Magnetic field strength is $4.0 \mathrm{kG}$. Sidearm temperature is $52^{\circ} \mathrm{C}$.

tron beam created plasma provides larger densities of high energy electrons, we believe this pumping scheme can be extended to a large variety of charge transfer laser systems with the potential for increased operating efficiency and output power.

\section{ACKNOWLEDGMENT}

The authors wish to thank B. Rosenvold and T. Burnell for skilled technical assistance.

\section{REFERENCES}

[1] J. J. Rocca, J. D. Meyer, Z. Yu, and G. J. Collins, presented at the 34th Gaseous Electron. Conf., Boston, MA, Oct. 1981.

[2] T. Shay, H. Kano, and G. J. Collins, Appl. Phys. Lett., vol. 26, p. 531, 1975; and Appl. Phys. Lett., vol. 29, p. 221, 1976.

[3] J. A. Piper, G. J. Collins, and C. E. Webb, Appl. Phys. Lett., vol. 21, p. $203,1972$.

[4] S. Hattori, H. Kano, K. Tokutome, G. J. Collins, and T. Goto, IEEE J. Quantum Electron., vol. QE-10, p. 530, 1974; and G. J. Collins, H. Kuno, S. Hattori, K. Tolutome, M. Ishikawa, and N. Kamiide, IEEE J. Quantum Electron., vol. QE-8, p. 679, 1972. 